\title{
Probing into the Recent Occurrence of Scrub Typhus Cases in and around Kolkata
}

Authors

\section{Dr Purba Mukherjee*1, Dr Atindra Kumar², Dr Shuvankar Mukherjee ${ }^{3}$, Dr Dibyendu Banerjee ${ }^{4}$}

${ }^{1}$ Assistant Professor, Department of Microbiology, Calcutta National Medical College, Kolkata ${ }^{2}$ Post Graduate Trainee ( $2^{\text {nd }}$ Year), Department of Microbiology, Calcutta National Medical College, Kolkata

${ }^{3}$ Assistant Professor, Department of Community Medicine, Calcutta National Medical College, Kolkata

${ }^{4}$ Associate Professor, Department of Microbiology, Calcutta National Medical College, Kolkata

*Corresponding Author

Dr Purba Mukherjee

\begin{abstract}
Scrub typhus is endemic in India. Right now scrub typhus is enjoying its status as a re-emerging disease and is often causing trouble in diagnosis due to absence of specific rash or eschar. In our institution, we observed a seasonal rise of febrile illnesses that coincided with monsoon rain, and decided to investigate the probable etiological role of scrub typhus among the undiagnosed fever cases. This prospective study was conducted from the middle of May to the end of August in 2019. All febrile patients who tested negative for malaria, dengue, urinary tract infections, enteric fever and other pyogenic infections were included in this study. Those who became serologically reactive for Scrub typhus IgM antibody by ELISA were considered as established cases of scrub typhus. The seroprevalence of scrub typhus among undiagnosed fever cases was $33.9 \%$ with a resultant mortality of $11.8 \%$. Fever (100\%), headache (92\%) and myalgia (62\%) were the commonest symptoms followed by lymphadenopathy, vomiting, diarrhea, pain abdomen, rash and rarely eschar. Meningoencephalitis (12\%) was the commonest complication followed by acute kidney injury which along with hepatitis often led to fatal multi organ failure. Thus, scrub typhus should be considered at the earliest in the differential diagnoses for acute undiagnosed fever with nonspecific features in endemic countries like ours.
\end{abstract}

Keywords: Scrub typhus, IgM ELISA, undiagnosed fever, non-specific features, meningoencephalitis.

\section{Introduction}

Scrub typhus is a well known zoonosis characterised by an acute febrile illness that can range from a self-limiting illness to fatal multiorgan failure. The disease is transmitted by bites of the 'chiggers', the larval stage of the trombiculid mite, which harbours the rickettsial pathogen Orientia tsutsugamushi ${ }^{[1]}$.
This febrile illness is endemic in a wide zone of the renowned 'Tsutsugamushi triangle' bounded by Japan in the east, Pakistan in the west, Russia in the north and northern Australia in the south; thus covering China, Korea, Indonesia, Malaysia, India and the islands of western Pacific and Indian ocean. However, recent reports have enlisted occurrences of scrub typhus in several regions outside this 
'infamous' triangle of rickettsial disease ${ }^{[2],[3]}$. India faced several outbreaks of scrub typhus since 1960s, the first reported cases being from Himachal Pradesh $^{[1]}$. Later on, the disease "virtually disappeared" due to use of insecticides for vector control, changes in lifestyle and empirical treatment of pyrexia with chloramphenicol and tetracycline group of drugs. The apparent well controlled status of the disease was enjoyed until recently when the disease reemerged in several regions of India namely Himachal Pradesh, Tamilnadu, Kerala, Karnataka, Madhya Pradesh, Jammu and Kashmir, Bihar, West Bengal, Uttaranchal, Rajasthan and Meghalaya $^{[1],[4]-[7]}$. West Bengal faced scrub typhus outbreak few years ago ${ }^{[8]}$. Still, there are less than required awareness about this potentially fatal zoonotic disease as aptly mentioned by WHO"Scrub typhus is probably one of the most underdiagnosed and under-reported febrile illnesses requiring hospitalization" $"$ [1].

In our institution, we have observed a seasonal rise of febrile illnesses coinciding with the monsoon rain who had negative laboratory reports for malaria, dengue, urinary tract infections and other pyogenic infections. Most of them presented with nonspecific flu-like symptoms with development of fatal complications like meningitis or multi organ failure (MOF) in certain percentage of patients. There were varied response with empirical antibiotics like doxycycline or azithromycin. Keeping in mind the endemicity of scrub typhus, we suspected that a silent outbreak of scrub typhus is going on in this particular area. We intended to evaluate the seroprevalence of scrub typhus $\operatorname{IgM}$ antibody by ELISA method which is considered as one of the confirmatory tests to detect acute scrub typhus disease ${ }^{[4]}$.

\section{Materials and Methods}

This prospective study was carried out from starting of May to the end of August, this year with rise in acute undiagnosed fever cases attending this tertiary care hospital catering primarily urban population. A detailed history taking and thorough clinical examination followed by laboratory screening was done for detection of malaria parasites, dengue, Japanese encephalitis, enteric fever, urinary tract infection and other pyogenic infections. Serum from patients who were negative for all those etiology were further evaluated for scrub typhus serology. All the serum samples were aliquoted and stored at $20^{\circ} \mathrm{C}$ till further testing. Scrub typhus IgM antibody was detected by using Scrub Typhus IgM ELISA kit (InBios) following the manufacturer's guidelines. The absorbance was measured with primary filter at $450 \mathrm{~nm}$ without any secondary filter. All the data were analysed methodically to determine statistical significance.

\section{Results}

In the last three and half months, 251 patients presented with febrile illnesses. Out of them, 85 were serologically confirmed by scrub typhus IgM ELISA with a seroprevalence of $33.9 \%$, having $53 \%$ males and $47 \%$ females among them. Among all the patients, $52 \%$ were from Kolkata whereas rest were mostly from semi-urban populations in the surrounding cities with scattered cases from some distal rural belts. (Table-1)

Fever and malaise for a duration of 5-14 days were the commonest findings found in majority of patients followed by headache, myalgia, lymphadenopathy, and gastrointestinal symptoms. Classical rash and eschar, the hallmarks of the disease, were rarely found, and till date we could not find any respiratory symptoms like cough or ARDS.(Table 2) Complications developed in 11 cases, with development of isolated meningoencephalitis in 3 cases. Multi organ failure occurred in 6 cases- presenting with any two of the followings- meningoencephalitis, acute kidney injury and hepatitis denoted by raised hepatic enzymes. Isolated fatal liver dysfunction was found in a solitary case. The overall mortality reached 11.8 $\%$ with CNS and renal involvement as the prime important prognostic markers. 
Table 1: Demographic characteristics of the scrub typhus reactive patients with percentage $(n=85)$

\begin{tabular}{|l|c|}
\hline Demographic characteristics & No. Of patients (\%) \\
\hline Regional distribution & $44(52)$ \\
\hline Kolkata & $41(48)$ \\
\hline Outside Kolkata \\
\hline Gender & $45(53)$ \\
\hline Male & $40(47)$ \\
\hline Female & $4(5)$ \\
\hline Age Groups & $28(33)$ \\
\hline 0-5yrs & $33(39)$ \\
\hline 6-18yrs & $12(14)$ \\
\hline 19-45 yrs & $8(9)$ \\
\hline 45-60 yrs & $19(22)$ \\
\hline$>60$ yrs & $58(68)$ \\
\hline Duration of illness & $8(9)$ \\
\hline$<5$ days & \\
\hline 5-14 days &
\end{tabular}

Table 2: Clinical presentations in Scrub typhus patients $(\mathrm{n}=85)$

\begin{tabular}{|l|c|}
\hline Clinical signs /symptoms & No. of patients (\%) \\
\hline Fever & $85(100)$ \\
\hline Malaise & $85(100)$ \\
\hline Headache & $78(92)$ \\
\hline Myalgia & $53(62)$ \\
\hline Lymphadenopathy & $43(50)$ \\
\hline Rash & $11(13)$ \\
\hline Eschar & $1(1.2)$ \\
\hline Vomiting & $49(58)$ \\
\hline Diarrhoea & $30(35)$ \\
\hline Pain abdomen & $20(24)$ \\
\hline Raised liver enzymes & $7(8)$ \\
\hline Acute kidney injury & $8(9)$ \\
\hline Seizures & $10(12)$ \\
\hline Meningitis & $10(12)$ \\
\hline Altered sensorium & $10(12)$ \\
\hline Multi organ failure (MOF) & $6(7)$ \\
\hline
\end{tabular}

\section{Discussion}

Scrub typhus can be designated as 'today's most important rickettsial disease, worldwide', accounting for up to $23 \%$ of all febrile episodes, with an occurrence of estimated 1 million cases annually while over one billion people are at risk. $^{[1],[2],[9]}$. Scrub typhus is an important reemerging pathogen in India as proven by several outbreaks reports in the last 10 years from various states like Tamil Nadu (2001-2002), Pondicherry (2008) Meghalaya (2010), Himachal Pradesh (2011), Nagaland $(2011)^{[8]}$. Indeed, it is the commonest rickettsial disease in the Indian subcontinent. In West Bengal, scrub typhus is endemic in the sub-Himalayan belt by rendering a favorable habitat to the mite-vector due to abundance of bushy vegetation, tea gardens, grassy fields and forests with adjacent cleared outskirts. Regular outbreaks were reported from Kurseong and Mirik in Darjeeling district of West Bengal (2005, 2010-2012) ${ }^{[10]}$. Recently, scrub typhus cases were reported from urban areas, too. Studies have pointed out important role of rodents in transmission of urban scrub typhus as well as role of various recreational activities like jogging, doing yoga or playing in a park, thus increasing the possibilities of mite or chigger bites ${ }^{[11]}$. In this background it took us some time to convince our clinicians that scrub typhus may be an important differential diagnosis in cases attending this urbanbased hospital with acute undiagnosed fever rapidly progressing to meningitis or fatal multi organ failure.

Starting from May, we observed a sharp rise of cases from the second half of June to the end of August, fairly coinciding with the monsoon rain. Similar seasonality of scrub typhus was observed by $\mathrm{R}$ Thomas and co-workers who commented that the increased humidity was favorable for hatching of mite eggs thus increasing the chances of chiggerbite $^{[12]}$. In South India, disease-peak was used to be observed in rainy and cooler months of the year ${ }^{[4]}$. $\mathrm{Li} \mathrm{T}$ et al in their study concluded that in a particular region, this seasonality was dependent on the climate, temperature and degree of rainfall in that very area ${ }^{[13]}$.

In our study, out of 85 confirmed cases, $53 \%$ were males and $47 \%$ females with a male preponderance of 1.1. GM Varghese and group found $48 \%$ males and $52 \%$ females in their study in South India ${ }^{[4]}$.

The majority of cases (52\%) were clustered in various urban slums in the metropolitan city of Kolkata, whereas cases from suburban and rural areas cumulatively were $48 \%$.

$86 \%$ cases occurred in 6- 60 years of age group, indicating involvement of active young and adult population, $5 \%$ cases in under 5 children and $9 \%$ cases in the geriatric age group. The patient 
population varied among the previous studies depending on the socio-demographical characteristics. A study from South India found mean age of patients $45+/-15$ years $^{[4]}$.

A diverse clinical spectrum was observed in our study. $92 \%$ of the febrile patients came with duration of illness between 5 days to 2 weeks. The common cluster of symptoms include fever, malaise, headache $(92 \%)$, myalgia $(62 \%)$, vomiting (58\%), lymphadenopathy (50\%) and diarrhoea $(35 \%)$. Pain abdomen (24\%) were commoner than rash $(13 \%)$. Eschar was found rarely $(1.2 \%)$. We have not seen cough or respiratory distress or development of ARDS in any of the cases. These findings were similar to that of other studies save respiratory involvement, for which they found cough and breathlessness in significant proportion of cases, ${ }^{[14],[15]}$, and eschar in a variable but higher proportion of cases ${ }^{[16]}$.

Case fatality rate was approximately $12 \%$ in our study. Studies from south India found a declining trend of mortality- from $14 \%$ in 2002-2003 to $9 \%$ in $2014^{[4],[17]}$. Studies from northern India pointed out a similar declining trend from $17.2 \%$ in 2004 to $14 \%$ in $2006^{[5],[18]}$.

Serious complications like meningoencephalitis, acute kidney injury, hepatitis were not uncommon in our study. Fatal fulminant hepatitis occurred in $1.2 \%$ cases. Acute kidney injury was found in $9 \%$ cases which is lower than that observed by Attur et $\mathrm{al}^{[19]}$. Isolated meningoencephalitis occurred in $6 \%$ in our study with a $60 \%$ fatality which was significantly higher than the non-meningitis group (p-value less than 0.05). Other studies found that CNS involvement in scrub typhus has shown an extended spectrum of manifestations commonest being meningitis, meningoencephalitis or encephalitis $^{[20]}$. Several studies in India pointed out the rate of occurrence of meningoencephalitis in scrub typhus in 23.3, 9.5 and 14 percent of cases $[4],[5],[15]$. Varghese et al. observed multi organ failure in a very high proportion $-34 \%$ of cases and pulmonary dysfunction as the commonest complication $(33.4 \%)$ followed by acute renal failure (18\%). They found a significantly higher mortality rate of $25 \%$ among the multi-organ failure group in comparison to that of $7 \%$ in patients without $\mathrm{MOF}^{[4]}$. On the other hand, we found only $7 \%$ of cases with MOF, but the relative mortality due to MOF was a striking $83 \%$ which means MOF a is significant prognostic marker with a $\mathrm{p}$-value of less than 0.05 .

\section{Conclusions}

Scrub typhus should be considered as a potentially fatal cause of acute febrile illnesses presenting with non- specific symptoms like fever, headache, myalgia and lymphadenopathy. GI symptoms like nausea, vomiting, diarrhoea, may be present in a fair number of cases. In endemic tropical countries like India, the eschar may not be present or even pass unnoticed. Similarly, rashes may be inconspicuous in dark skinned people if not absent.

Febrile illness that leads to newly onset seizures or altered sensorium or acute encephalitis syndrome may have scrub encephalitis. So, in all non-JE AES cases the possible role of scrub typhus should also be ruled out by ELISA or other confirmatory tests. As it is a potentially fatal disease which rapidly turns into complications like multi-organ failure, high degree of clinical suspicion followed by laboratory confirmation may lead to significant reduction in mortality and morbidity from this disease.

\section{Acknowledgement}

We thankfully acknowledge Dr. P P Mukherjee, Dean of Students Affairs, Professor and HOD, Department of Medicine, and Dr. R Guhathakurta, Professor and HOD, Department of Mictobiology for their kind cooperation.

\section{Conflicts of interest: None declared}

\section{References}

1. Chunchanur Sk. Scrub Typhus in India- an impending threat! Ann Clin Immunol Microbiol. 2018;1,(1):1003 tropical med 3010011 
2. Ju Jiang, Allen L. Richards. Scrub Typhus: No longer restricted to the Tsutsugamushi triangle. Trop Med Infect Dis. 2018,3,11; doi: 10.3390/tropicalmed3010018

3. Alison Luce-Fedrow, Marcie L.Lehman, Daryl J. Kelly, et al. A Review of Scrub Typhus (Orientiatsutsugamushi and Related Organisms): Then, Now and Tomorrow. Trop Med Infect Dis. 2018, 3(1),8; doi:10.3390/tropicalmed3010008

4. Varghese GM, Trowbridge P, Janardhanan J, et al. Clinical profile and improving mortality trend of scrub typhus in South India. Int J Infect Dis. 2014 Jun;23:3943.doi:10.1016/j.ijid.2014.02.009.Epub 2014 Mar 21

5. Mahajan SK, Rolain JM, Kashyap R, et al. Scrub typhus in Himalayas. Emerg Infect Dis. 2006;12: 1590-2

6. Chrispal A, Boorugu H, Gopinath $\mathrm{KG}$ et al. Scrub typhus: an unrecognised threat in South India- clinical profile and predictors of mortality. Trop Doc.2010;40: 129-33

7. Batra HV. Spotted fever and typhus fever in Tamil Nadu. Indian J Med Res. 2007; 126: 101-3

8. Chakraborty S, Sarma N. Scrub typhus: An emerging threat. Indian J Dermatol. 2017; 62: 478-85

9. Watt G., Parola P, Scrub typhus and tropical rickettsioses. Curr Opin infect Dis. 2003;16: 429-436

10. Sharma PK, Ramakrishnan R, Hutin YJ, et al. Scrub typhus in Darjeeling, India: Opportunities for simple, practical prevention measures. Trans R Soc Trop Med Hyg. 2009; 103v:1153-8.

11. Ray Laskar A, Suri S, Acharya AS. Scrub Typhus: Re-emerging Public Health Problem in India. J Commun. Dis. 2015; 47 (3):19-25.

12. Thomas R, Puranik P, Kalal B et al. Fie-year analysis of rickettsial fevers in children in South India: Clinical manifestations and complications, J Infect Dev Ctries.2016;10 (6): 657-61

13. Li T, Yang Z, Dong Z, et al. Meteorological factors and risk of scrub typhus in Guangzhou, sothern China, 2006-12. BMC infect Dis.2014; 14:139

14. Mathai E, Rolain JM, Verghese GM, et al. Outbreak of Scrub typhus in Southern India during cooler months. Ann NY Acad Sci.20003;990:359-64

15. Vivekanandan M, Mani A, Priya YS et al. Outbreak of scrub typhus in Pondicherry. J Assoc Physicians India.2010; 58: 24-8

16. Kim DM, Won KJ, Park CY et al. Distribution of eschars on the body of scrub typhus patients: a prospective study. Am J Trop Med Hyg.2007;76:806-9

17. Varghese GM, Abraham OC, Mathai D, et al. Scrub typhus among hospitalised patients with febrile illness in South India: magnitude and clinical predictors. J Infect.2006; 52:56-60

18. Kumar K, Saxena VK, Thomas TG, et al. Outbreak investigation of scrub typhus in Himachal Pradesh (India). J Commun Dis 2004; 36:277-83

19. Attur RP, Kuppasami S, Bairy M, et al. Acute kidney injury in scrub typhus. ClinExpNephrol. 2013; 17:725-29

20. Peter JV, Sudarshan TI, Prakash JAJ, et al. Severe scrub typhus infection: Clinical features, diagnostic challenges and management. World J Crit Care Med. 2015 Aug 4; 4(3): 244-250. 